\title{
Cordial elements and dimensions of affine Deligne-Lusztig varieties
}

\author{
Xuhua $\mathrm{He}$ \\ The Institute of Mathematical Sciences and Department of Mathematics, The Chinese University of Hong Kong, Shatin, N.T., \\ Hong Kong SAR, China; E-mail: xuhuahe@math.cuhk.edu.hk.
}

Received: 9 July 2020; Revised: 25 May 2021; Accepted: 10 August 2021

2020 Mathematics Subject Classification: Primary - 11G25; Secondary - 20G25.

\begin{abstract}
The affine Deligne-Lusztig variety $X_{w}(b)$ in the affine flag variety of a reductive group $\mathbf{G}$ depends on two parameters: the $\sigma$-conjugacy class $[b]$ and the element $w$ in the Iwahori-Weyl group $\tilde{W}$ of $\mathbf{G}$. In this paper, for any given $\sigma$-conjugacy class $[b]$, we determine the nonemptiness pattern and the dimension formula of $X_{w}(b)$ for $\operatorname{most} w \in \tilde{W}$.
\end{abstract}

\section{Introduction}

\subsection{Motivation}

The notion of the affine Deligne-Lusztig variety was introduced by Rapoport in [Ra05]. It plays an important role in arithmetic geometry and the Langlands programme. One of the main motivations comes from the reduction of Shimura varieties. In this paper we focus on the affine Deligne-Lusztig varieties in the affine flag variety. In this case, the affine Deligne-Lusztig varieties are closely related to the Shimura varieties with Iwahori level structure. On the special fibres, there are two important stratifications:

- Newton stratification, indexed by specific $\sigma$-conjugacy classes $[b]$ in the associated $p$-adic group

○ Kottwitz-Rapoport stratification, indexed by specific elements $w$ in the associated Iwahori-Weyl group

A fundamental question is to determine when the intersection of a Newton stratum indexed by $[b]$ and a Kottwitz-Rapoport stratum indexed by $w$ is nonempty and to determine its dimension. Such an intersection is closely related to the affine Deligne-Lusztig variety $X_{w}(b)$ (see, e.g., [HR17]). In a parallel story over function fields, affine Deligne-Lusztig varieties also arise naturally in the study of local shtukas (see, e.g., [HV11]).

Motivated by the study of Shimura varieties and local shtukas, we would like to understand the following fundamental questions on affine Deligne-Lusztig varieties:

$\circ$ When is the affine Deligne-Lusztig variety nonempty?

- If nonempty, what is its dimension?

It is also worth pointing out that much information on affine Deligne-Lusztig varieties in partial affine flag varieties (which are closely related to Shimura varieties with other parahoric level 
structures) can be deduced from the information on affine Deligne-Lusztig varieties in the affine flag variety.

\subsection{The main result}

In this paper we determine, for any given $\sigma$-conjugacy class $[b]$, the nonemptiness pattern and dimension formula of $X_{w}(b)$ for most $w$ in the Iwahori-Weyl group $\tilde{W}$. To state the result, we introduce some notation first. For simplicity, we will consider here only the split groups $\mathbf{G}$. The general case will be studied in the body of the paper.

The Iwahori-Weyl group $\tilde{W}$ is the semidirect product of the coweight lattice with the relative Weyl group $W_{0}$. We may write $\tilde{W}$ as $\tilde{W}=\sqcup_{\lambda}$ is dominant $W_{0} t^{\lambda} W_{0}$. For any $w \in W_{0} t^{\lambda} W_{0}$, we set $\lambda_{w}=\lambda$. The $\sigma$-conjugacy classes [b] are classified by Kottwitz [Ko85] via two invariants: the image under the Kottwitz map $\kappa$ and the Newton point $v_{b}$ (which is a dominant rational coweight). By Mazur's inequality for affine Deligne-Lusztig varieties in the affine Grassmannian [Ga10], we deduce that if $X_{w}(b) \neq \emptyset$, then $\kappa(w)=\kappa(b)$ and $\lambda_{w} \geq v_{b}$ with respect to the dominance order of the rational coweights.

The converse, however, is far from being true. The main result of this paper is the following:

Theorem 1.1. Let $w \in \tilde{W}$. Suppose that $w$ is in a shrunken Weyl chamber. If $\kappa(w)=\kappa(b), \lambda_{w}-v_{b}$ is a linear combination of the simple coroots with all the coefficients positive and $\lambda_{w}^{b b} \geq v_{b}$, then we have a complete description of the nonemptiness pattern and dimension formula for $X_{w}(b)$.

We refer to Section 2.2 for the definition of shrunken Weyl chambers, Section 5.2 for the definition of $-^{b b}$ and Theorem 6.1 for the precise description of the nonemptiness pattern and dimension formula. These assumptions are satisfied for example when $\lambda_{w} \geq v_{b}+2 \rho^{\vee}$, where $\rho^{\vee}$ is the half sum of positive coroots (see Corollary 6.4).

As an application of Theorem 1.1, in joint work with Q. Yu [HY21] we establish a dimension formula for the group-theoretic analogue of Newton strata for sufficiently large dominant coweights.

\subsection{Some previous results}

In [GHKR10, Conjecture 9.5.1], Görtz, Haines, Kottwitz and Reuman made several influential conjectures on the nonemptiness pattern and dimension formula of $X_{w}(b)$.

First, for the basic $\sigma$-conjugacy class $[b]$, they gave a conjecture in [GHKR10, Conjecture 9.5.1 (a)] on the nonemptiness pattern and dimension formula of $X_{w}(b)$ for $w$ in the shrunken Weyl chamber. This conjecture was established in [He14]. For $X_{w}(b)$ with [b] basic and $w$ outside the shrunken Weyl chamber, in [GHKR10, Conjecture 9.4.2] they gave a conjecture on the nonemptiness pattern. This conjecture is established in [GHN15]. But for [b] basic and $w$ outside the shrunken Weyl chamber, no conjectural dimension formula of $X_{w}(b)$ has even been formulated so far.

For arbitrary $\sigma$-conjugacy class $[b]$, they made an interesting conjecture in [GHKR10, Conjecture 9.5.1 (b)] which predicts the difference of the dimensions of $X_{w}(b)$ and $X_{w}\left(b_{\text {basic }}\right)$, where $\left[b_{\text {basic }}\right.$ ] is the unique basic $\sigma$-conjugacy class such that $\kappa(b)=\kappa\left(b_{\text {basic }}\right)$. In this conjecture, $w$ is not required to be shrunken, but the length of $w$ is required to be big enough with some (unspecified) lower bound. In later works, we studied $X_{w}(b)$ via a somewhat different direction. First, the assumption that $w$ is in the shrunken Weyl chamber is added, as even for the basic $b$, the dimension formula of $X_{w}(b)$ with $w$ outside the shrunken Weyl chamber is still very mysterious. Second, we would like to have a specific lower bound on $w$.

For split groups and the case where $[b]$ is represented by translation elements, under the "very shrunken' assumption the nonemptiness pattern and the dimension formula of $X_{w}(b)$ were given in [He15, Theorem $2.28 \&$ Theorem 2.34]. A similar result was obtained in [MST19] under a different condition on $w$. 
For other nonbasic $\sigma$-conjugacy classes, little is known so far on the nonemptiness pattern and dimension formula of $X_{w}(b)$.

\subsection{Old strategies}

We discuss several strategies used in previous work to study the nonemptiness pattern and dimension formula for $X_{w}(b)$.

The emptiness pattern is established via the method of $P$-alcove elements introduced in [GHKR10, Definition 2.1.1]. The upper bound of $\operatorname{dim} X_{w}(b)$ is given by the virtual dimension $d_{w}(b)$ introduced in $[\mathrm{He} 14, \S 10.1]$.

In [He14], we combined the Deligne-Lusztig reduction with some remarkable properties of minimal length elements in their conjugacy classes in $\tilde{W}$ to establish a method to compute $\operatorname{dim} X_{w}(b)$ for arbitrary $w$ and arbitrary $[b]$. As a consequence, in [He14, Theorem 6.1] we established the 'dimension=degree' theorem, which relates the dimension of affine Deligne-Lusztig varieties with the degree of the class polynomials of the affine Hecke algebras. However, the computation of the class polynomials, in general, is extremely difficult. The dimension=degree theorem does not lead to explicit descriptions of the nonemptiness pattern and the dimension formula of $X_{w}(b)$.

For basic $[b]$, assume that $X_{w}(b) \neq \emptyset$. It remains to show that $\operatorname{dim} X_{w}(b)$ reaches the upper bound $d_{w}(b)$. Note that for any Coxeter element $c, \operatorname{dim} X_{c}(b)$ is easy to compute. This will be used as the starting point. In $[\mathrm{He} 14, \S 11]$, we constructed an explicit 'reduction path' from an element $w$ in the shrunken Weyl chamber to an element $w^{\prime}$ with finite part a Coxeter element. By [HY12, Theorem 1.1], the minimal length elements in the conjugacy class of $w^{\prime}$ in $\tilde{W}$ are the Coxeter elements $c$. This gives a reduction path from $w$ to $c$ and thus leads to a lower bound of $\operatorname{dim} X_{w}(b)$. Fortunately, the lower bound also equals the virtual dimension $d_{w}(b)$. Thus we proved the nonemptiness pattern and the dimension formula of $X_{w}(b)$ with basic $[b]$.

For split groups and the case where $[b]$ is represented by translation elements, in [He15] we used the superset method of [GHKR10] to relate the nonemptiness pattern and dimension formula of $X_{w}(b)$ with $X_{w^{\prime}}(1)$ for a given $w^{\prime}$. Note that [1] is a basic $\sigma$-conjugacy class. We then used the result on $X_{w^{\prime}}(1)$ established in [He14] to obtain the desired result on $X_{w}(b)$. A very different approach was introduced in [MST19], where the authors used alcove walks and Littelmann paths to study the nonemptiness pattern and dimension formula of $X_{w}(b)$.

It is unclear how or whether the methods in [He15] or in [MST19] for the translation elements may be generalised to arbitrary $\sigma$-conjugacy classes $[b]$. The reduction method introduced in [He14] works, in theory, for an arbitrary $\sigma$-conjugacy class $[b]$. However, constructing an explicit reduction path from a given $w$ to a minimal length element associated to a nonbasic $[b]$ is very challenging. Q. Yu has written a computer program to construct the reduction path for groups with small ranks. But so far it is not clear how such a reduction path may be constructed in general.

\subsection{New strategy}

The new strategy in this paper is as follows. Instead of using minimal length elements as the starting point, we use the cordial elements introduced by Milićević and Viehmann in [MV20] as the starting point. In Section 4, we construct a new family of cordial elements. For any element $w^{\prime}$ in this family, $\operatorname{dim} X_{w^{\prime}}(b)$ equals the virtual dimension. We then construct in Section 5 an explicit reduction path from an element $w$ in the shrunken Weyl chamber to an element in this family. This is where the assumption $\lambda_{w}^{b b} \geq v_{b}$ is used. This shows that $\operatorname{dim} X_{w}(b) \geq d_{w}(b)$. Finally we use the result that $\operatorname{dim} X_{w}(b) \leq d_{w}(b)$ established in [He14] to prove the desired nonemptiness pattern and the dimension formula of $X_{w}(b)$.

Another issue we would like to point out here is that previous works (e.g., [GH10]) are in less general situations (e.g., with the assumption that $G$ is split or tamely ramified) than the one we consider here. However, in this paper we use the geometric results Theorem 3.2 and Proposition 3.3, which hold for any reductive group $G$, and then use combinatorics of the Iwahori-Weyl groups $\tilde{W}$. The results from 
previous works that we use here are to deduce certain nice combinatorial properties of $\tilde{W}$. Thus we may apply the previous works in the more general setup here.

\section{Preliminaries}

\subsection{The reductive group $\mathrm{G}$ and its Iwahori-Weyl group}

Let $F$ be a nonarchimedean local field and $\breve{F}$ be the completion of the maximal unramified extension of $F$. We write $\Gamma$ for Gal $(\bar{F} / F)$, where $\bar{F}$ is an algebraic closure of $F$. We write $\Gamma_{0}$ for the inertia subgroup of $\Gamma$. Let $t$ be a uniformiser in $F$.

Let $\mathbf{G}$ be a connected reductive group over $F$. Let $\sigma$ be the Frobenius morphism of $\breve{F} / F$. We write $\breve{G}$ for $\mathbf{G}(\breve{F})$. We use the same symbol $\sigma$ for the induced Frobenius morphism on $\breve{G}$.

We fix a maximal $\breve{F}$-split torus $S$ in $\mathbf{G}$ defined over $F$ which contains a maximal $F$-split torus. Let $T$ be the centraliser of $S$ in $\mathbf{G}$. Then $T$ is a maximal torus. Let $\mathcal{A}$ be the apartment of $\mathbf{G}_{\breve{F}}$ corresponding to $S_{\breve{F}}$. Thus $\mathcal{A}$ is (noncanonically) isomorphic to $V=X_{*}(T)_{\Gamma_{0}} \otimes_{\mathbb{Z}} \mathbb{R}$. The Frobenius $\sigma$ naturally acts on $\mathcal{A}$. We fix a $\sigma$-stable alcove $\mathfrak{a}$ in $\mathcal{A}$, and let $\breve{I} \subset \breve{G}$ be the Iwahori subgroup corresponding to $\mathfrak{a}$. Thus $\breve{I}$ is $\sigma$-stable.

We denote by $N$ the normaliser of $T$ in $\mathbf{G}$. The relative Weyl group $W_{0}$ is defined to be $N(\breve{F}) / T(\breve{F})$. The Iwahori-Weyl group (associated to $S$ ) is defined as

$$
\tilde{W}=N(\breve{F}) / T(\breve{F}) \cap \breve{I} .
$$

For any $w \in \tilde{W}$, we choose a representative $\dot{w}$ in $N(L)$.

We have a natural short exact sequence $0 \rightarrow X_{*}(T)_{\Gamma_{0}} \rightarrow \tilde{W} \rightarrow W_{0} \rightarrow 0$. We choose a special vertex of $\mathfrak{a}$ and represent $\tilde{W}$ as a semidirect product,

$$
\tilde{W}=X_{*}(T)_{\Gamma_{0}} \rtimes W_{0}=\left\{t^{\lambda} w ; \lambda \in X_{*}(T)_{\Gamma_{0}}, w \in W_{0}\right\} .
$$

The Iwahori-Weyl group $\tilde{W}$ contains the affine Weyl group $W_{a}$ as a normal subgroup and we have

$$
\tilde{W}=W_{a} \rtimes \Omega,
$$

where $\Omega$ is the stabiliser of $\mathfrak{a}$. The length function $\ell$ and Bruhat order $\leq$ on $W_{a}$ extend in a natural way to $\tilde{W}$. The Frobenius $\sigma$ naturally acts on $\tilde{W}$, in such a way that the subset $\tilde{\mathbb{S}} \subset \tilde{W}$ is stable.

For any $K \subset \tilde{\mathbb{S}}$, we denote by $W_{K}$ the subgroup of $\tilde{W}$ generated by $s \in K$. Let ${ }^{K} \tilde{W}$ (resp., $\tilde{W}^{K}$ ) be the set of minimal length elements in their cosets in $W_{K} \backslash \tilde{W}$ (resp., $\tilde{W} / W_{K}$ ).

Let $\mathbb{S} \subset \tilde{\mathbb{S}}$ be the set of simple reflections of $W_{0}$. By convention, the dominant Weyl chamber of $V$ is opposite to the unique Weyl chamber containing $\mathfrak{a}$. Let $\Delta$ be the set of relative simple roots determined by the dominant Weyl chamber. For any $s \in \mathbb{S}$, we denote by $\alpha_{s} \in \Delta$ the corresponding simple root and $\alpha_{s}^{\vee}$ the corresponding simple coroot. We denote by $w_{\mathbb{S}}$ the longest element of $W_{0}$.

We define the $\sigma$-conjugation action on $\breve{G}$ by $g \cdot{ }_{\sigma} g^{\prime}=g g^{\prime} \sigma(g)^{-1}$. Let $B(\mathbf{G})$ be the set of $\sigma$-conjugacy classes on $\breve{G}$. The classification of the $\sigma$-conjugacy classes was obtained by Kottwitz in [Ko85]. Any $\sigma$-conjugacy class $[b]$ is determined by two invariants:

$\circ$ the element $\kappa([b]) \in \Omega_{\sigma}$ and

$\circ$ the Newton point $v_{b} \in\left(\left(X_{*}(T)_{\Gamma_{0}, \mathbb{Q}}\right)^{+}\right)^{\langle\sigma\rangle}$.

Here $-{ }_{\sigma}$ denotes the $\sigma$-coinvariants and $\left(X_{*}(T)_{\Gamma_{0}, \mathbb{Q}}\right)^{+}$denotes the set of dominant elements in $X_{*}(T)_{\Gamma_{0}} \otimes \mathbb{Q}=X_{*}(T)^{\Gamma_{0}} \otimes \mathbb{Q}$; the action of $\sigma$ on $\left(X_{*}(T)_{\Gamma_{0}, \mathbb{Q}}\right) / W_{0}$ is transferred to an action on $\left(X_{*}(T)_{\mathbb{Q}}\right)^{+}$ (L-action).

For any $w \in \tilde{W}$, we write $\kappa(w)$ for $\kappa(\dot{w})$. It is easy to see that $\kappa(w)$ is independent of the choice of the representative $w$. 
We use the convention of Bruhat and Tits that the translation element $t^{\lambda}$ acts by $-\lambda$ on the apartment. In this way, we have $\ell\left(x t^{\lambda}\right)=\ell(x)+\ell\left(t^{\lambda}\right)$ for any $x \in W_{0}$ and $\lambda$ dominant.

\subsection{Affine Deligne-Lusztig varieties}

We have the following generalisation of the Bruhat decomposition:

$$
\breve{G}=\sqcup_{w \in \tilde{W}} \breve{I} \dot{w} \breve{I}
$$

due to Iwahori and Matsumoto [IM65] in the split case and to Bruhat and Tits [BT72] in the general case. Let $F l=\breve{G} / \breve{I}$ be the affine flag variety. For any $b \in \breve{G}$ and $w \in \tilde{W}$, we define the corresponding affine Deligne-Lusztig variety in the affine flag variety:

$$
X_{w}(b)=\left\{g \breve{I} \in \breve{G} / \breve{I} ; g^{-1} b \sigma(g) \in \breve{I} \dot{w} \breve{I}\right\} \subset F l .
$$

In the equal characteristic, $X_{w}(b)$ is the set of $\overline{\mathbb{F}}_{q}$-points of a scheme [BS17].

As discussed in [GHN15, §2], the study of the nonemptiness pattern and dimension formula of affine Deligne-Lusztig varieties for an arbitrary reductive group may be reduced to simple and quasi-split groups over $F$. From now on, we assume that $\mathbf{G}$ is simple and quasi-split over $F$. In this case, the $\sigma$-action on $\tilde{W}$ preserves $W_{0}$ and $X_{*}(T)_{\Gamma_{0}}$. Moreover, we have $\sigma(\mathbb{S})=\mathbb{S}$ and $\sigma(\Delta)=\Delta$.

Now we recall the definition of the virtual dimension in [He14, §10.1].

Note that any element $w \in \tilde{W}$ may be written in a unique way as $w=x t^{\mu} y$ with $\mu$ dominant, $x, y \in W_{0}$, such that $t^{\mu} y \in{ }^{\mathbb{S}} \tilde{W}$. In this case,

$$
\ell(w)=\ell(x)+\ell\left(t^{\mu}\right)-\ell(y) .
$$

We set

$$
\eta_{\sigma}(w)=\sigma^{-1}(y) x .
$$

Let $\mathbf{J}_{b}$ be the reductive group over $F$ with $\mathbf{J}_{b}(F)=\left\{g \in \breve{G} ; g b \sigma(g)^{-1}=b\right\}$. The defect of $b$ is defined by $\operatorname{def}(b)=\operatorname{rank}_{F} \mathbf{G}-\operatorname{rank}_{F} \mathbf{J}_{b}$. Here for a reductive group $\mathbb{H}$ defined over $F, \operatorname{rank}_{F}$ is the $F$-rank of the group $\mathbb{H}$. Let $\rho$ be the dominant weight with $\left\langle\alpha^{\vee}, \rho\right\rangle=1$ for any $\alpha \in \Delta$. The virtual dimension is defined to be

$$
d_{w}(b)=\frac{1}{2}\left(\ell(w)+\ell\left(\eta_{\sigma}(w)\right)-\operatorname{def}(b)\right)-\left\langle v_{b}, \rho\right\rangle .
$$

The following result is proved in [He14, Corollary 10.4] for residually split groups and in [He15, Theorem 2.30] for the general case:

Theorem 2.1. Let $b \in \breve{G}$ and $w \in \tilde{W}$. Then $\operatorname{dim} X_{w}(b) \leq d_{w}(b)$.

For any $w \in W_{0}$, we denote by $\operatorname{supp}(w) \subset \mathbb{S}$ the set of simple reflections appearing in some (or equivalently, any) reduced expression of $w$. We set $\operatorname{supp}_{\sigma}(w)=\cup_{i \in \mathbb{Z}} \sigma^{i}(\operatorname{supp}(w))$.

For any $w \in \tilde{W}$, let $\lambda_{w}$ be the unique dominant coweight such that $w \in W_{0} t^{\lambda_{w}} W_{0}$. For any $\lambda \in X_{*}(T)_{\Gamma_{0}}$, we denote by $\lambda^{\diamond}$ the average of the $\sigma$-orbit of $\lambda$. For any $\lambda, \lambda^{\prime} \in X_{*}(T)_{\mathbb{Q}}^{+}$, we write $\lambda \geq \lambda^{\prime}$ if $\lambda-\lambda^{\prime} \in \sum_{\alpha \in \Delta} \mathbb{Q}_{\geq 0} \alpha^{\vee}$ and write $\lambda \geq_{\mathbb{Z}} \lambda^{\prime}$ if $\lambda-\lambda^{\prime} \in \sum_{\alpha \in \Delta} \mathbb{N} \alpha^{\vee}$. Here $\mathbb{N}$ is the set of natural numbers, that is, the set of nonnegative integers.

A critical strip of the apartment $V$ is the subset $\{v ;-1<\langle v, \alpha\rangle<0\}$ for a given positive root $\alpha$ in the reduced root system associated to the affine Weyl group $W_{a}$. We remove all the critical strips from $V$ and call each connected component of the remaining subset of $V$ a shrunken Weyl chamber. 


\section{Some combinatorial properties}

\subsection{Minimal length elements}

For any $\sigma$-conjugacy class $\mathcal{O}$ in $\tilde{W}$, we denote by $\mathcal{O}_{\text {min }}$ the set of minimal length elements in $\mathcal{O}$. For $w, w^{\prime} \in \tilde{W}$ and $s \in \tilde{\mathbb{S}}$, we write $w \stackrel{s}{\rightarrow} \sigma w^{\prime}$ if $w^{\prime}=\operatorname{sw} \sigma(s)$ and $\ell\left(w^{\prime}\right) \leq \ell(w)$. We write $w \rightarrow \sigma w^{\prime}$ if there is a sequence $w=w_{0}, w_{1}, \ldots, w_{n}=w^{\prime}$ of elements in $\tilde{W}$ such that for any $k, w_{k-1} \stackrel{s}{\rightarrow} \sigma w_{k}$ for some $s \in \tilde{\mathbb{S}}$. We write $w \approx_{\sigma} w^{\prime}$ if $w \rightarrow_{\sigma} w^{\prime}$ and $w^{\prime} \rightarrow_{\sigma} w$. It is easy to see that $w \approx_{\sigma} w^{\prime}$ if $w \rightarrow_{\sigma} w^{\prime}$ and $\ell(w)=\ell\left(w^{\prime}\right)$.

The following result is proved in [HN14, §2]:

Theorem 3.1. Let $\mathcal{O}$ be a $\sigma$-conjugacy class of $\tilde{W}$ and $w \in \mathcal{O}$. Then there exists $w^{\prime} \in \mathcal{O}_{\min }$ such that $w \rightarrow \sigma w^{\prime}$.

Theorem 3.2. Let $b \in \breve{G}$ and $w \in \mathcal{O}_{\min }$ for some $\sigma$-conjugacy class $\mathcal{O}$ of $\tilde{W}$. Then $X_{w}(b) \neq \emptyset$ if and only if $\dot{w} \in[b]$. In this case, $\operatorname{dim} X_{w}(b)=\ell(w)-\left\langle v_{b}, 2 \rho\right\rangle$.

\subsection{Deligne-Lusztig reduction}

Now we recall the 'reduction' à la Deligne and Lusztig for affine Deligne-Lusztig varieties (see [DL76, Proof of Theorem 1.6] and [GH10, Corollary 2.5.3]).

Proposition 3.3. Let $b \in \breve{G}$. Then

(1) if $w, w^{\prime} \in \tilde{W}$ with $w \approx_{\sigma} w^{\prime}$, we have

$$
\operatorname{dim} X_{w}(b)=\operatorname{dim} X_{w^{\prime}}(b)
$$

(2) if $w \in \tilde{W}$ and $s \in \tilde{\mathbb{S}}$ with $\ell(\operatorname{sw\sigma } \sigma(s))=\ell(w)-2$, we have

$$
\operatorname{dim} X_{w}(b)=\max \left\{\operatorname{dim} X_{s w}(b), \operatorname{dim} X_{s w \sigma(s)}(b)\right\}+1 .
$$

Here, by convention, we set $\operatorname{dim} \emptyset=-\infty$ and $-\infty+n=-\infty$ for any $n \in \mathbb{R}$.

\subsection{The relation $\Rightarrow$}

Following [GH10, Definition 3.1.4], for $w, w^{\prime} \in \tilde{W}$ we write $w \Rightarrow_{\sigma} w^{\prime}$ if for any $b \in \breve{G}$,

$$
\operatorname{dim} X_{w}(b)-d_{w}(b) \geq \operatorname{dim} X_{w^{\prime}}(b)-d_{w^{\prime}}(b) .
$$

Again by convention, we set $\operatorname{dim} \emptyset=-\infty$. If the right-hand side is $-\infty$, then the inequality holds regardless of the left-hand side. It is also easy to see that the relation is transitive.

Note that by the definition of virtual dimension, $w \Rightarrow_{\sigma} w^{\prime}$ if and only if for any $b \in \breve{G}$ with $X_{w^{\prime}}(b) \neq \emptyset, X_{w}(b) \neq \emptyset$, and in this case,

$$
\operatorname{dim} X_{w}(b)-\operatorname{dim} X_{w^{\prime}}(b) \geq \frac{1}{2}\left(\ell(w)+\ell\left(\eta_{\sigma}(w)\right)-\ell\left(w^{\prime}\right)-\ell\left(\eta_{\sigma}\left(w^{\prime}\right)\right)\right) .
$$

We write $w \Leftrightarrow_{\sigma} w^{\prime}$ if $w \Rightarrow_{\sigma} w^{\prime}$ and $w^{\prime} \Rightarrow_{\sigma} w$.

\subsection{The monoid structure on $\tilde{W}$}

By [He09, Lemma 1], for any $w, w^{\prime} \in \tilde{W}$ the subset $\left\{u w^{\prime} ; u \leq w\right\}$ of $\tilde{W}$ contains a unique maximal element which we denote by $w * w^{\prime}$. Moreover, $w * w^{\prime}=\max \left\{u v ; u \leq w, v \leq w^{\prime}\right\}$. Hence $*$ is associative. This gives a monoid structure on $\tilde{W}$. If $w_{1} \leq w$ and $w_{1}^{\prime} \leq w^{\prime}$, then $w_{1} * w_{1}^{\prime} \leq w * w^{\prime}$. 


\section{The cordial elements}

\subsection{Definition}

There is a natural partial ordering $\leq$ on $B(\mathbf{G})$ defined as follows: Set $[b],\left[b^{\prime}\right] \in B(\mathbf{G})$. Then $[b] \leq\left[b^{\prime}\right]$ if $\kappa(b)=\kappa\left(b^{\prime}\right)$ and $v_{b} \leq v_{b^{\prime}}$.

Now we recall the cordial elements introduced by Milićević and Viehmann in [MV20].

For any $w \in \tilde{W}$, there is a unique maximal $\sigma$-conjugacy class $[b]$ such that $X_{w}(b) \neq \emptyset$. We denote this $\sigma$-conjugacy class by $\left[b_{w}\right]$. The element $w$ is called cordial if $\operatorname{dim} X_{w}\left(b_{w}\right)=d_{w}\left(b_{w}\right)$. Equivalently, $w$ is cordial if and only if $\ell(w)-\ell\left(\eta_{\sigma}(w)\right)=\left\langle v_{b_{w}}, 2 \rho\right\rangle-\operatorname{def}\left(b_{w}\right)$ [MV20, Definition 3.14].

By definition, if $w \Leftrightarrow \sigma w^{\prime}$, then $w$ is a cordial element if and only if $w^{\prime}$ is a cordial element. The following result is proved in [MV20, Theorem $1.1 \&$ Corollary 3.17]:

Theorem 4.1. Let $w \in \tilde{W}$ be a cordial element. Then the following hold:

(1) Set $[b],\left[b^{\prime}\right] \in B(\mathbf{G})$. If $[b] \leq\left[b^{\prime}\right] \leq\left[b_{w}\right]$ and $X_{w}(b) \neq \emptyset$, then $X_{w}\left(b^{\prime}\right) \neq \emptyset$.

(2) If $X_{w}(b) \neq \emptyset$, then $\operatorname{dim} X_{w}(b)=d_{w}(b)$.

It is mentioned in [MV20] that fully characterising the cordial elements is fairly difficult. In [MV20, Theorem 1.2], some interesting families of cordial elements are provided. The main result of this section is to provide another family of cordial elements.

Theorem 4.2. Let $\lambda$ be a dominant coweight and set $x \in W_{0}$. Then $x t^{\lambda}$ is a cordial element and $\left[b_{x t^{\lambda}}\right]=\left[\dot{t}^{\lambda}\right]$.

Remark 4.3. The original proof we had was a bit technical. The proof to come was suggested by E. Viehmann.

\subsection{Mazur's inequality}

Recall that $\mathbf{G}$ is quasi-split over $F$. Let $\breve{K} \supset \breve{I}$ be a $\sigma$-stable special maximal parahoric subgroup of $\breve{G}$. The nonemptiness pattern of the affine Deligne-Lusztig varieties in the affine Grassmannian $\breve{G} / \breve{K}$ is determined in terms of Mazur's inequality. This was established by Gashi [Ga10, Theorem 1.1] for unramified groups and proved in the general case in [He14, Theorem 7.1]. We may reformulate the result as follows:

Theorem 4.4. Let $\lambda$ be a dominant coweight and set $b \in \breve{G}$. Then $[b] \cap \breve{K} \dot{t}^{\lambda} \breve{K} \neq \emptyset$ if and only if $\kappa(b)=\kappa\left(t^{\lambda}\right)$ and $v_{b} \leq \lambda^{\diamond}$.

\subsection{Proof of Theorem 4.2}

Set $w \in \tilde{W}$. By definition, $\left[b_{w}\right]$ is the unique maximal $\sigma$-conjugacy class that intersects $\breve{I} \dot{w} \breve{I}$. By [Vi14, Corollary 5.6], $\left[b_{w}\right]$ is also the unique maximal $\sigma$-conjugacy class that intersects $\breve{I} \dot{\mathscr{w}} \breve{I}$. Since $t^{\lambda} \leq x t^{\lambda} \leq w_{\mathbb{S}} t^{\lambda}$, we have

$$
\breve{I t^{\lambda}} \breve{I} \subset \overline{\breve{I} \dot{x} \dot{t}^{\lambda} \breve{I}} \subset \bar{I} \dot{w}_{\mathbb{S}} \dot{t}^{\lambda} \breve{I}=\overline{\breve{K} \dot{t}^{\lambda} \breve{K}}
$$

By Theorem 4.4, $\left[b_{w_{\mathbb{S}} t^{\lambda}}\right]=\left[\dot{t}^{\lambda}\right]$. Thus $\left[b_{x t^{\lambda}}\right]=\left[\dot{t}^{\lambda}\right]$.

Now $v_{b_{x t^{\lambda}}}=\lambda^{\diamond}$ and $\operatorname{def}\left(b_{x t^{\lambda}}\right)=0$. Hence

$$
\ell\left(x t^{\lambda}\right)-\ell\left(\eta_{\sigma}\left(x t^{\lambda}\right)\right)=\ell(x)+\ell\left(t^{\lambda}\right)-\ell(x)=\ell\left(t^{\lambda}\right)=\langle\lambda, 2 \rho\rangle=\left\langle\lambda^{\diamond}, 2 \rho\right\rangle .
$$

Thus $x t^{\lambda}$ is a cordial element. 


\subsection{Another family of cordial elements}

Set $w \in \tilde{W}$ such that $w \mathfrak{a}$ is in the dominant Weyl chamber-that is, $w=w_{\mathbb{S}} t^{\lambda} y$, where $\lambda$ is a dominant coweight and $y \in W_{0}$ with $t^{\lambda} y \in{ }^{\mathbb{S}} \tilde{W}$. It was proved by Milićević and Viehmann in [MV20, Theorem 1.2 (a)] that $w$ is also a cordial element.

Now we show that it can also be deduced from Theorem 4.2.

Set $w^{\prime}=\sigma^{-1}(y) w_{\mathbb{S}} t^{\lambda}$. By Theorem 4.2, $w^{\prime}$ is a cordial element. Note that $\eta_{\sigma}\left(w^{\prime}\right)=\eta_{\sigma}(w)=$ $\sigma^{-1}(y) w_{\mathbb{S}}$. Moreover, it is easy to see that $w \approx_{\sigma} w^{\prime}$. Hence $w \Leftrightarrow_{\sigma} w^{\prime}$, and $w$ is also a cordial element.

It is also worth mentioning that not every element of the form $x t^{\lambda}$ is $\approx_{\sigma}$-equivalent to an element in the dominant Weyl chamber.

\section{From $w$ to a cordial element}

We first show the following:

Proposition 5.1. Let $\lambda, \lambda^{\prime}$ be dominant coweights. Then the set

$$
\left\{\mu^{\prime} ; \mu^{\prime} \text { is dominant, } \mu^{\prime}+\lambda^{\prime} \geq_{\mathbb{Z}} \lambda\right\}
$$

contains a unique minimal element with respect the dominance order $\geq_{\mathbb{Z}}$.

Remark 5.2. The proof is due to $\mathrm{S}$. Nie.

Proof. Let $\mu_{1}^{\prime}, \mu_{2}^{\prime}$ be dominant coweights with $\mu_{1}^{\prime}+\lambda^{\prime} \geq_{\mathbb{Z}} \lambda$ and $\mu_{2}^{\prime}+\lambda^{\prime} \geq_{\mathbb{Z}} \lambda$. We may write $\mu_{1}^{\prime}-\mu_{2}^{\prime}$ as $\mu_{1}^{\prime}-\mu_{2}^{\prime}=\gamma_{1}-\gamma_{2}$, where $\gamma_{1} \in \sum_{\alpha \in J_{1}} \mathbb{Z}_{>0} \alpha, \gamma_{2} \in \sum_{\alpha \in J_{2}} \mathbb{Z}_{>0} \alpha$ for some $J_{1}, J_{2} \subset \Delta$ with $J_{1} \cap J_{2}=\emptyset$.

Set $\mu=\mu_{1}^{\prime}-\gamma_{1}=\mu_{2}^{\prime}-\gamma_{2}$. Set $\alpha \in \Delta$. Since $J_{1} \cap J_{2}=\emptyset$, we have $\alpha \notin J_{1}$ or $\alpha \notin J_{2}$. If $\alpha \notin J_{1}$, then $\langle\mu, \alpha\rangle \geq\left\langle\mu_{1}^{\prime}, \alpha\right\rangle \geq 0$. If $\alpha \notin J_{2}$, then $\langle\mu, \alpha\rangle \geq\left\langle\mu_{2}^{\prime}, \alpha\right\rangle \geq 0$. Thus $\mu$ is dominant. By definition, $\mu_{1}^{\prime} \geq_{\mathbb{Z}} \mu$ and $\mu_{2}^{\prime} \geq_{\mathbb{Z}} \mu$. Moreover,

$$
\lambda^{\prime}-\lambda+\mu_{1}^{\prime}=\lambda^{\prime}-\lambda+\mu_{2}^{\prime}+\gamma_{1}-\gamma_{2} \in\left(\sum_{\alpha \in \Delta} \mathbb{Z}_{>0} \alpha+\gamma_{1}-\gamma_{2}\right) \cap \sum_{\alpha \in \Delta} \mathbb{Z}_{>0} \alpha .
$$

Since $J_{1} \cap J_{2}=\emptyset$, we have $\lambda^{\prime}-\lambda+\mu_{1}^{\prime}-\gamma_{1} \in \sum_{\alpha \in \Delta} \mathbb{Z}_{>0} \alpha$. In other words, $\lambda^{\prime}+\mu \geq_{\mathbb{Z}} \lambda$.

The statement is proved.

\subsection{The normalised subtraction}

For any dominant coweights $\lambda, \lambda^{\prime}$, we denote by $\lambda-_{\text {dom }} \lambda^{\prime}$ the unique minimal element in the set

$$
\left\{\mu^{\prime} ; \mu^{\prime} \text { is dominant, } \mu^{\prime}+\lambda^{\prime} \geq_{\mathbb{Z}} \lambda\right\} .
$$

It is easy to see that if $\lambda-\lambda^{\prime}$ is dominant, then $\lambda-_{\text {dom }} \lambda^{\prime}=\lambda-\lambda^{\prime}$. We call $-_{\text {dom }}$ the normalised subtraction. Now we prove some of its properties.

Corollary 5.3. Let $\lambda, \lambda^{\prime}$ be dominant coweights. Let $\lambda^{\prime \prime}$ be a dominant coweight with $\lambda^{\prime} \geq_{\mathbb{Z}} \lambda^{\prime \prime}$. Set $x \in W_{0}$ and let $\mu$ be the unique dominant coweight in the $W_{0}$-orbit of $\lambda-x\left(\lambda^{\prime \prime}\right)$. Then $\mu \geq_{\mathbb{Z}} \lambda-\operatorname{dom} \lambda^{\prime}$.

Proof. Note that $\mu-\left(\lambda-x\left(\lambda^{\prime \prime}\right)\right) \in \sum_{\alpha \in \Delta} \mathbb{N} \alpha, \lambda^{\prime \prime}-x\left(\lambda^{\prime \prime}\right) \in \sum_{\alpha \in \Delta} \mathbb{N} \alpha$ and $\lambda^{\prime}-\lambda^{\prime \prime} \in \sum_{\alpha \in \Delta} \mathbb{N} \alpha$. Thus

$$
\begin{aligned}
\mu+\lambda^{\prime} & =\left(\mu-\lambda+x\left(\lambda^{\prime \prime}\right)\right)+\lambda-x\left(\lambda^{\prime \prime}\right)+\lambda^{\prime} \\
& =\left(\mu-\lambda+x\left(\lambda^{\prime \prime}\right)\right)+\left(\lambda^{\prime \prime}-x\left(\lambda^{\prime \prime}\right)\right)+\left(\lambda^{\prime}-\lambda^{\prime \prime}\right)+\lambda \\
& \geq_{\mathbb{Z}} \lambda .
\end{aligned}
$$


Corollary 5.4. Let $\lambda, \lambda_{1}, \lambda_{2}$ be dominant coweights. Then

$$
\left(\lambda-{ }_{\text {dom }} \lambda_{1}\right)-{ }_{\text {dom }} \lambda_{2}=\lambda-{ }_{\text {dom }}\left(\lambda_{1}+\lambda_{2}\right) .
$$

Proof. Set $\mu_{1}=\left(\lambda-_{\text {dom }} \lambda_{1}\right)-_{\text {dom }} \lambda_{2}$ and $\mu_{2}=\lambda-_{\text {dom }}\left(\lambda_{1}+\lambda_{2}\right)$. By definition,

$$
\left(\lambda_{1}+\lambda_{2}\right)+\mu_{1}=\lambda_{1}+\left(\lambda_{2}+\mu_{1}\right) \geq_{\mathbb{Z}} \lambda_{1}+\left(\lambda-\operatorname{dom} \lambda_{1}\right) \geq_{\mathbb{Z}} \lambda .
$$

So $\mu_{1} \geq_{\mathbb{Z}} \mu_{2}$.

On the other hand,

$$
\lambda_{1}+\left(\lambda_{2}+\mu_{2}\right)=\left(\lambda_{1}+\lambda_{2}\right)+\mu_{2} \geq_{\mathbb{Z}} \lambda
$$

So by definition, $\lambda_{2}+\mu_{2} \geq_{\mathbb{Z}} \lambda-_{\text {dom }} \lambda_{1}$ and $\mu_{2} \geq_{\mathbb{Z}} \mu_{1}$.

\subsection{The double flat operator}

For any subset $J$ of $\mathbb{S}$, we denote by $\rho_{J}^{\vee}$ the dominant coweight with

$$
\left\langle\rho_{J}^{\vee}, \alpha_{s}\right\rangle= \begin{cases}1 & \text { if } s \in J, \\ 0 & \text { if } s \notin J .\end{cases}
$$

Let $\eta_{J}^{\vee}$ be the unique dominant coweight in the $W_{0}$-orbit of $-\sigma^{-1}\left(\rho_{J}^{\vee}\right)$.

Set $w \in \tilde{W}$. We write $w$ as $w=x t^{\lambda} y$ with $\lambda$ dominant, $x, y \in W_{0}$ and $t^{\lambda} y \in{ }^{\mathbb{S}} \tilde{W}$. Let $J=\{s \in$ $\mathbb{S} ; s y<y\}$. Since $t^{\lambda} y \in{ }^{\mathbb{S}} \tilde{W}$, we have $\left\langle\lambda, \alpha_{s}\right\rangle>0$ for any $s \in J$. In particular, $\lambda-\rho_{J}^{\vee}$ is dominant. We set

$$
\lambda_{w}^{b b}=\left(\lambda-\rho_{J}^{\vee}\right)-_{\operatorname{dom}} \eta_{J}^{\vee}=\lambda-_{\operatorname{dom}}\left(\rho_{J}^{\vee}+\eta_{J}^{\vee}\right) .
$$

The main result of this section is as follows:

Theorem 5.5. Assume that $\mathbf{G}$ is quasi-split over F. Set $w \in \tilde{W}$ such that wa is in a shrunken Weyl chamber. Then there exist a dominant coweight $\gamma$ with $\gamma \geq_{\mathbb{Z}} \lambda_{w}^{b b}$ and $a \in W_{0}$ with $\operatorname{supp}_{\sigma}(a) \supset$ $\operatorname{supp}_{\sigma}\left(\eta_{\sigma}(w)\right)$ such that

$$
w \Rightarrow_{\sigma} a t^{\gamma}
$$

\subsection{A convenient notation}

Following [GH10, §2.4], we give a convenient notation for varieties of tuples of elements in $F l$. We explain the notation by examples. Let $\mathcal{O}_{w}=\{(g \breve{I}, g \dot{w} \breve{I}) ; g \in \breve{G}\} \subset F l \times F l$. Then we set

$$
\left\{g \stackrel{w}{\longrightarrow} g^{\prime \prime} \stackrel{w^{\prime}}{\longrightarrow} g^{\prime}\right\}=\left\{\left(g, g^{\prime}, g^{\prime \prime}\right) \in(F l)^{3} ;\left(g, g^{\prime \prime}\right) \in \mathcal{O}_{w},\left(g^{\prime \prime}, g^{\prime}\right) \in \mathcal{O}_{w^{\prime}}\right\}
$$

Similarly,

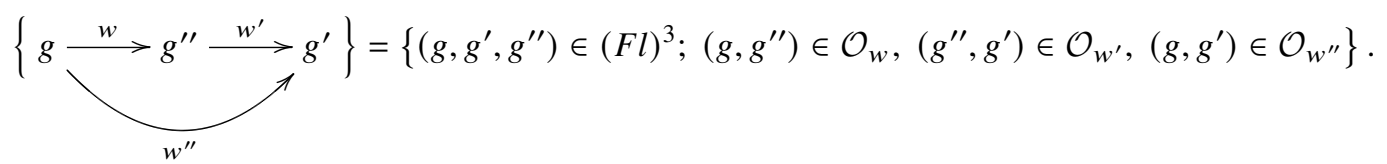

The affine Deligne-Lusztig varieties can be written as

$$
X_{w}(b)=\{g \stackrel{w}{\longrightarrow} b \sigma(g)\} .
$$


In all these cases, we do not distinguish between the sets given by the conditions on the relative position and the corresponding locally closed sub-ind-schemes of the product of affine flag varieties. The following result is proved in [GH10, Proposition 2.5.2]:

Proposition 5.6. Set $w, w^{\prime} \in \tilde{W}$, and set $w^{\prime \prime} \in\left\{w w^{\prime}, w * w^{\prime}\right\}$. Then the map

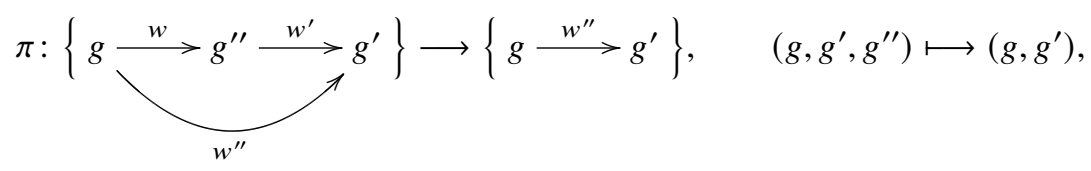

is surjective. Moreover, all the fibres have dimension

$$
\operatorname{dim} \pi^{-1}\left(\left(g, g^{\prime}\right)\right) \geq \begin{cases}\ell(w)+\ell\left(w^{\prime}\right)-\ell\left(w * w^{\prime}\right) & \text { if } w^{\prime \prime}=w * w^{\prime} \\ \frac{1}{2}\left(\ell(w)+\ell\left(w^{\prime}\right)-\ell\left(w w^{\prime}\right)\right) & \text { if } w^{\prime \prime}=w w^{\prime}\end{cases}
$$

\subsection{Proof of Theorem 5.5}

We write $w$ as $w=x t^{\lambda_{w}} y$ with $x, y \in W_{0}$ and $t^{\lambda_{w}} y \in{ }^{\mathbb{S}} \tilde{W}$. Let $J=\{s \in \mathbb{S} ; s y<y\}$ and $J^{\prime}=$ $\left\{s \in \mathbb{S} ; s\left(\lambda_{w}-\rho_{J}^{\vee}\right)=\lambda_{w}-\rho_{J}^{\vee}\right\}$. We write $\sigma^{-1}(y) x$ as $\sigma^{-1}(y) x=x^{\prime} z$ for some $x^{\prime} \in W_{0}^{J^{\prime}}$ and $z \in W_{J^{\prime}}$. By [GH10, §2.3], the assumption that $w \mathfrak{a}$ is in a shrunken Weyl chamber implies that $x \alpha_{j}<0$ for any $j \in J^{\prime}$. In particular,

$$
\ell\left(x z^{-1}\right)=\ell(x)-\ell(z)
$$

Let $\gamma$ be the unique dominant coweight in the $W_{0}$-orbit of $\lambda_{w}-\rho_{J}^{\vee}+\left(x^{\prime}\right)^{-1} \sigma^{-1}\left(\rho_{J}^{\vee}\right)$. By Corollary 5.3, $\gamma \geq_{\mathbb{Z}} \lambda_{w}^{b b}$. Let $K=\{s \in \mathbb{S} ; s(\gamma)=\gamma\}$ and set $y^{\prime} \in W_{0}^{K}$ with $\lambda_{w}-\rho_{J}^{\vee}+\left(x^{\prime}\right)^{-1} \sigma^{-1}\left(\rho_{J}^{\vee}\right)=y^{\prime}(\gamma)$.

Set $\alpha>0$ with $\left(y^{\prime}\right)^{-1} \alpha<0$. Then $\left\langle\gamma,\left(y^{\prime}\right)^{-1} \alpha\right\rangle \leq 0$. On the other hand, if $\left\langle\gamma,\left(y^{\prime}\right)^{-1} \alpha\right\rangle=0$, then since $y^{\prime} \in W_{0}^{K}$, we have $\alpha=y^{\prime}\left(\left(y^{\prime}\right)^{-1} \alpha\right)<0$. That is a contradiction. Hence $\left\langle y^{\prime}(\gamma), \alpha\right\rangle=\left\langle\gamma,\left(y^{\prime}\right)^{-1} \alpha\right\rangle<0$. Since $\lambda_{w}-\rho_{J}^{\vee}$ is dominant, $\left\langle\lambda_{w}-\rho_{J}^{\vee}, \alpha\right\rangle \geq 0$. Thus $\left\langle\left(x^{\prime}\right)^{-1} \sigma^{-1}\left(\rho_{J}^{\vee}\right), \alpha\right\rangle=\left\langle\sigma^{-1}\left(\rho_{J}^{\vee}\right), x^{\prime}(\alpha)\right\rangle<0$. Since $\sigma^{-1}\left(\rho_{J}^{\vee}\right)$ is dominant, $x^{\prime}(\alpha)<0$. By [GH10, Lemma 2.6.1], we have

$$
\ell\left(x^{\prime} y^{\prime}\right)=\ell\left(x^{\prime}\right)-\ell\left(y^{\prime}\right) \text {. }
$$

Set $s \in J^{\prime}$. Since $x^{\prime} \in W_{0}^{J^{\prime}}$, we have $\ell\left(x^{\prime} s\right)=\ell\left(x^{\prime}\right)+1$. Thus $\ell\left(x^{\prime}\right)-\ell\left(y^{\prime}\right)=\ell\left(x^{\prime} y^{\prime}\right)=\ell\left(\left(x^{\prime} s\right)\left(s y^{\prime}\right)\right) \geq$ $\ell\left(x^{\prime} s\right)-\ell\left(s y^{\prime}\right)=\ell\left(x^{\prime}\right)+1-\ell\left(s y^{\prime}\right)$. So $\ell\left(s y^{\prime}\right) \geq \ell\left(y^{\prime}\right)+1$. Therefore

$$
y^{\prime} \in{ }^{J^{\prime}} W_{0} .
$$

In particular, we have

$$
\ell\left(\left(y^{\prime}\right)^{-1} z\right)=\ell\left(y^{\prime}\right)+\ell(z)
$$

Set $w_{1}=x z^{-1} t^{\lambda_{w}-\rho_{J}^{\vee}} y^{\prime}$ and $w_{2}=\left(y^{\prime}\right)^{-1} z t^{\rho_{J}^{\vee}} y$. Then $w=w_{1} w_{2}$. By formula (5.3), $t^{\lambda_{w}-\rho_{J}^{\vee}} y^{\prime} \in{ }^{\mathbb{S}} \tilde{W}$. By the definition of $J$, we have $t^{\rho_{J}^{\vee}} y \in \mathbb{S} \tilde{W}$. Hence by equation (2.1), we have

$$
\begin{gathered}
\ell(w)=\ell(x)+\ell\left(t^{\lambda_{w}}\right)-\ell(y) \\
\ell\left(w_{1}\right)=\ell\left(x z^{-1}\right)+\ell\left(t^{\lambda_{w}-\rho_{J}^{\vee}}\right)-\ell\left(y^{\prime}\right) \\
\ell\left(w_{2}\right)=\ell\left(\left(y^{\prime}\right)^{-1} z\right)+\ell\left(t^{\rho_{J}^{\vee}}\right)-\ell(y) .
\end{gathered}
$$


By equations (5.1) and (5.4), we have

$$
\begin{aligned}
\ell\left(w_{1}\right)+\ell\left(w_{2}\right) & =\ell(x)-\ell(z)+\ell\left(t^{\lambda_{w}-\rho_{J}^{\vee}}\right)-\ell\left(y^{\prime}\right)+\ell\left(y^{\prime}\right)+\ell(z)+\ell\left(t^{\rho_{J}^{\vee}}\right)-\ell(y) \\
& =\ell(x)+\ell\left(t^{\lambda_{w}}\right)-\ell(y)=\ell(w) .
\end{aligned}
$$

By equations (5.2) and (5.4), we have

$$
\begin{aligned}
\ell\left(\left(y^{\prime}\right)^{-1} z\right)+\ell\left(x^{\prime} y^{\prime}\right) & =\ell\left(y^{\prime}\right)+\ell(z)+\ell\left(x^{\prime}\right)-\ell\left(y^{\prime}\right)=\ell\left(x^{\prime}\right)+\ell(z) \\
& =\ell\left(x^{\prime} z\right)=\ell\left(\sigma^{-1}(y) x\right)
\end{aligned}
$$

By equation (5.8) we have

$$
X_{w}(b)=\{g \stackrel{w}{\longrightarrow} b \sigma(g)\} \cong\left\{g \stackrel{w_{1}}{\longrightarrow} g_{1} \stackrel{w_{2}}{\longrightarrow} b \sigma(g)\right\} .
$$

Set

$$
X_{1}=\left\{g_{1} \stackrel{w_{2}}{\longrightarrow} g_{2} \stackrel{\sigma\left(w_{1}\right)}{\longrightarrow} b \sigma\left(g_{1}\right)\right\} \cong\left\{g_{1} \stackrel{\left(y^{\prime}\right)^{-1} z}{\longrightarrow} g_{3} \stackrel{t^{\rho_{J}} y}{\longrightarrow} g_{2} \stackrel{\sigma\left(w_{1}\right)}{\longrightarrow} b \sigma\left(g_{1}\right)\right\} .
$$

Here the isomorphism follows from equation (5.7).

The map $\left(g, g_{1}\right) \mapsto\left(g_{1}, b \sigma(g)\right)$ is a universal homeomorphism from $X_{w}(b)$ to $X_{1}$. We have $y \sigma\left(x z^{-1}\right)=\sigma\left(\sigma^{-1}(y) x z^{-1}\right)=\sigma\left(x^{\prime}\right)$ and

$$
t^{\rho_{J}^{\vee}} y \sigma\left(w_{1}\right)=t^{\rho_{J}^{\vee}} \sigma\left(x^{\prime} t^{\lambda_{w}-\rho_{J}^{\vee}} y^{\prime}\right)=\sigma\left(x^{\prime}\right) \sigma\left(t^{y^{\prime}(\gamma)}\right) \sigma\left(y^{\prime}\right)=\sigma\left(x^{\prime} y^{\prime} t^{\gamma}\right) .
$$

Let

$$
X_{2}=\left\{g_{1} \stackrel{\left(y^{\prime}\right)^{-1} z}{\longrightarrow} g_{3} \stackrel{t^{\rho_{J}^{\vee} y}}{\longrightarrow} g_{2} \stackrel{\sigma\left(w_{1}\right)}{\longrightarrow} b \sigma\left(g_{1}\right)\right\} \subset X_{1} .
$$

We have

$$
\operatorname{dim}\left(X_{w}(b)\right)=\operatorname{dim}\left(X_{1}\right) \geq \operatorname{dim}\left(X_{2}\right) .
$$

Since $\gamma$ is dominant, we have $\ell\left(\sigma\left(x^{\prime} y^{\prime} t^{\gamma}\right)\right)=\ell\left(\sigma\left(x^{\prime} y^{\prime}\right)\right)+\ell\left(\sigma\left(t^{\gamma}\right)\right)$. Set

$$
X_{3}=\left\{g_{1} \stackrel{\left(y^{\prime}\right)^{-1} z}{\longrightarrow} g_{3} \stackrel{\sigma\left(x^{\prime} y^{\prime} t^{\gamma}\right)}{\longrightarrow} b \sigma\left(g_{1}\right)\right\} \cong\left\{g_{1} \stackrel{\left(y^{\prime}\right)^{-1} z}{\longrightarrow} g_{3} \stackrel{\sigma\left(x^{\prime} y^{\prime}\right)}{\longrightarrow} g_{4} \stackrel{\sigma\left(t^{\gamma}\right)}{\longrightarrow} b \sigma\left(g_{1}\right)\right\} .
$$

Let $\pi: X_{2} \rightarrow X_{3}$ be the projection map. Set $N_{1}=\frac{\ell\left(t^{\rho_{J}^{\vee} y}\right)+\ell\left(w_{1}\right)-\ell\left(x^{\prime} y^{\prime} t^{\gamma}\right)}{2}$. By Proposition 5.6, the map $\pi$ is surjective and the dimension of each fibre is larger than or equal to $N_{1}$.

We show that

(a) $\operatorname{dim}\left(X_{2}\right) \geq \operatorname{dim}\left(X_{3}\right)+N_{1}$.

Now suppose that $\operatorname{dim}\left(X_{2}\right)<\operatorname{dim}\left(X_{3}\right)+N_{1}$. Let $Z$ be an irreducible component of $X_{3}$ with $\operatorname{dim} Z=$ $\operatorname{dim} X_{3}$. For each irreducible component $Y$ of $\pi^{-1}(Z)$, we construct a closed subscheme $Z_{Y}$ of $Z$ such that $\operatorname{dim}\left(\pi^{-1}(z) \cap Y\right)<N_{1}$ if $z \in Z-Z_{Y}$. The construction is as follows. 
If $\pi(Y)$ is not dense in $Z$, then let $Z_{Y}$ be the closure of $\pi(Y)$. If $\pi(Y)$ is dense in $Z$, then the morphism $\pi: Y \rightarrow Z$ is dominant. By [GW10, Corollary 14.116], there exists an open dense subscheme $V$ of $Z$ contained in $\pi(Y)$ such that for any $z \in V$, we have $\operatorname{dim}\left(\pi^{-1}(z) \cap Y\right)=\operatorname{dim}(Y)-\operatorname{dim}(Z) \leq$ $\operatorname{dim}\left(X_{2}\right)-\operatorname{dim}\left(X_{3}\right)<N_{1}$. We set $Z_{Y}=Z-V$. This finishes our construction.

Note that in either case, $Z_{Y}$ is a proper subscheme of $Z$. Hence $\cup_{Y} Z_{Y} \varsubsetneqq Z$. Set $z \in Z-\cup_{Y} Z_{Y}$. Then $\operatorname{dim}\left(\pi^{-1}(z) \cap Y\right)<N_{1}$ for any irreducible component $Y$ of $\pi^{-1}(Z)$. Thus $\operatorname{dim}\left(\pi^{-1}(z)\right)<N_{1}$. That gives a contradiction.

So (a) is proved.

Set $a=\sigma^{-1}\left(\left(y^{\prime}\right)^{-1} z\right) *\left(x^{\prime} y^{\prime}\right)$. Then

$$
\begin{aligned}
\operatorname{supp}_{\sigma}(a) & =\operatorname{supp}_{\sigma}(\sigma(a))=\operatorname{supp}_{\sigma}\left(\left(y^{\prime}\right)^{-1} z\right) \cup \operatorname{supp}_{\sigma}\left(\sigma\left(x^{\prime} y^{\prime}\right)\right) \\
& =\operatorname{supp}_{\sigma}\left(\left(y^{\prime}\right)^{-1} z\right) \cup \operatorname{supp}_{\sigma}\left(x^{\prime} y^{\prime}\right) \\
& \supset \operatorname{supp}_{\sigma}\left(x^{\prime} y^{\prime}\left(y^{\prime}\right)^{-1} z\right)=\operatorname{supp}_{\sigma}\left(x^{\prime} z\right) \\
& =\operatorname{supp}_{\sigma}\left(\eta_{\sigma}(w)\right) .
\end{aligned}
$$

We set

$$
\begin{array}{r}
X_{4}=\left\{g_{1} \stackrel{\left(y^{\prime}\right)^{-1} z}{\longrightarrow} g_{3} \stackrel{\sigma\left(x^{\prime} y^{\prime}\right)}{\longrightarrow} g_{4} \stackrel{\sigma\left(t^{\gamma}\right)}{\longrightarrow} b \sigma\left(g_{1}\right)\right\} \subset X_{3}, \\
X_{5}=\left\{g_{1} \stackrel{\sigma(a)}{\longrightarrow} g_{4} \stackrel{\sigma\left(t^{\gamma}\right)}{\longrightarrow} b \sigma\left(g_{1}\right)\right\} .
\end{array}
$$

By Proposition 5.6 and the same argument as in (a),

$$
\operatorname{dim}\left(X_{3}\right) \geq \operatorname{dim}\left(X_{5}\right)+\ell\left(\left(y^{\prime}\right)^{-1} z\right)+\ell\left(x^{\prime} y^{\prime}\right)-\ell(a)=\operatorname{dim}\left(X_{5}\right)+\ell\left(\eta_{\sigma}(w)\right)-\ell(a) .
$$

Notice that $\ell\left(\sigma\left(a t^{\gamma}\right)\right)=\ell(\sigma(a))+\ell\left(\sigma\left(t^{\gamma}\right)\right)$. Thus the map $\left(g_{1}, g_{4}\right) \mapsto g_{1}$ gives an isomorphism $X_{5} \cong X_{\sigma\left(a t^{\gamma}\right)}(b)$, which is universally homeomorphic to $X_{a t^{\gamma}}(b)$. If $X_{a t^{\gamma}}(b) \neq \emptyset$, then $X_{\sigma\left(a t^{\gamma}\right)}(b) \neq \emptyset$ and $X_{w}(b) \neq \emptyset$. Note that $\ell\left(\left(y^{\prime}\right)^{-1} z\right)+\ell\left(x^{\prime} y^{\prime}\right)=\ell\left(\sigma^{-1}(y) x\right)=\ell\left(\eta_{\sigma}(w)\right)$. Therefore,

$$
\begin{aligned}
\operatorname{dim} & X_{w}(b)-\operatorname{dim} X_{a t^{\gamma}}(b) \\
\geq & \frac{\ell\left(t^{\rho_{J}^{\vee}} y\right)+\ell\left(w_{1}\right)-\ell\left(x^{\prime} y^{\prime} t^{\gamma}\right)}{2}+\ell\left(\eta_{\sigma}(w)\right)-\ell(a) \\
& =\frac{\ell\left(t^{\rho_{J}^{\vee}} y\right)+\ell\left(\left(y^{\prime}\right)^{-1} z\right)+\ell\left(w_{1}\right)-\ell\left(x^{\prime} y^{\prime} t^{\gamma}\right)+\ell\left(x^{\prime} y^{\prime}\right)-\ell(a)}{2}+\frac{\ell\left(\eta_{\sigma}(w)\right)}{2}-\frac{\ell(a)}{2} \\
& =\frac{\ell\left(w_{2}\right)+\ell\left(w_{1}\right)-\ell\left(a t^{\gamma}\right)}{2}+\frac{\ell\left(\eta_{\sigma}(w)\right)}{2}-\frac{\ell(a)}{2} \\
& =\frac{\ell(w)-\ell\left(a t^{\gamma}\right)}{2}+\frac{\ell\left(\eta_{\sigma}(w)\right)}{2}-\frac{\ell(a)}{2}=d_{w}(b)-d_{a t^{\gamma}}(b) .
\end{aligned}
$$

So $w \Rightarrow_{\sigma} a t^{\gamma}$. The theorem is proved. 


\section{Proof of main theorem}

Now we state our main result.

Theorem 6.1. Suppose that $\mathbf{G}$ is quasi-split over $F$. Set $b \in \breve{G}$ and $w \in \tilde{W}$ such that $w \mathfrak{a}$ is in a shrunken Weyl chamber, $\lambda_{w}^{\diamond}-v_{b} \in \sum_{\alpha \in \Delta} \mathbb{Q}_{>0} \alpha^{\vee}$ and $\left(\lambda_{w}^{b b}\right)^{\diamond} \geq v_{b}$. Then $X_{w}(b) \neq \emptyset$ if and only if $\kappa(b)=\kappa(w)$ and $\operatorname{supp}_{\sigma}\left(\eta_{\sigma}(w)\right)=\mathbb{S}$. In this case, $\operatorname{dim} X_{w}(b)=d_{w}(b)$.

Remark 6.2. It is worth mentioning that in most cases, $\lambda_{w}-\lambda_{w}^{b b}$ is dominant and nonzero. In this case, $\lambda_{w}^{\diamond}-\left(\lambda_{w}^{b b}\right)^{\diamond} \in \sum_{\alpha \in \Delta} \mathbb{Q}_{>0} \alpha^{\vee}$. However, if $\mathbf{G}$ is split over $F$ and $\lambda_{w}$ is a minuscule coweight, then $\lambda_{w}^{b b}=\lambda_{w}$. Thus the assumption $\lambda_{w}^{\diamond}-v_{b} \in \sum_{\alpha \in \Delta} \mathbb{Q}_{>0} \alpha^{\vee}$ is needed in our statement.

We first prove the theorem and then discuss the assumptions in the statement. In particular, we will give a simple condition where the assumptions are satisfied in Corollary 6.4.

\subsection{The $(J, w, \delta)$-alcove elements}

We recall the alcove elements introduced in [GHKR10] for split groups and then generalised to quasisplit groups in [GHN15].

For any $J \subset \mathbb{S}$ with $\sigma(J)=J$, we denote by $\mathbb{M}_{J} \subset \mathbf{G}$ the standard Levi subgroup corresponding to $J$ and let $\mathbb{P}_{J} \supset \mathbb{M}_{J}$ be the standard parabolic subgroup. Let $\mathbb{U}_{\mathbb{P}_{J}}$ be the unipotent radical of $\mathbb{P}_{J}$.

Set $J \subset \mathbb{S}$ with $\sigma(J)=J$ and $x \in W_{0}$. Set $w \in \tilde{W}$. We say that $w$ is a $(J, x, \sigma)$-alcove element if $x^{-1} w \sigma(x) \in \tilde{W}_{J}$ and ${ }^{\dot{x}} \mathbb{U}_{\mathbb{P}_{J}}(\breve{F}) \cap \dot{w} \breve{I} \subseteq{ }^{\dot{x}} \mathbb{U}_{\mathbb{P}_{J}}(\breve{F}) \cap \breve{I}$. The following result is proved in [GHN15, Corollary 3.6.1]. ${ }^{1}$

Theorem 6.3. Set $[b] \in B(\mathbf{G})$ and $w \in \tilde{W}$. Suppose that $w$ is a $(J, x, \sigma)$-alcove element. Let $\kappa_{\mathbb{M}_{J}}$ be the Kottwitz map for the group $\mathbb{M}_{J}$. If $\kappa_{\mathbb{M}_{J}}\left(x^{-1} w \sigma(x)\right) \neq \kappa_{\mathbb{M}_{J}}\left(b^{\prime}\right)$ for any $b^{\prime} \in[b] \cap \mathbb{M}_{J}(\breve{F})$, then $X_{w}(b)=\emptyset$.

\subsection{The emptiness pattern}

Suppose that $w \mathfrak{a}$ is in a shrunken Weyl chamber and $\left(\lambda_{w}^{b}\right)^{\diamond} \geq v_{b}$. We write $w$ as $w=x t^{\lambda} y$ with $x, y \in W_{0}$ and $t^{\lambda} y \in{ }^{\mathbb{S}} \tilde{W}$. If $\kappa(b) \neq \kappa(w)$, then $X_{w}(b)=\emptyset$.

Now suppose that $\kappa(b)=\kappa(w)$ and $\operatorname{supp}_{\sigma}\left(\sigma^{-1}(y) x\right) \neq \mathbb{S}$. Set $J=\operatorname{supp}_{\sigma}\left(\sigma^{-1}(y) x\right)$. By [GHN15, Lemma 3.6.3], $w$ is a $\left(J, \sigma^{-1}(y), \sigma\right)$-alcove element. Set $b^{\prime} \in[b] \cap \mathbb{M}_{J}(\breve{F})$. We denote by $v_{b^{\prime}}^{\mathbb{M}_{J}}$ the image of $b^{\prime}$ under the Newton map for $\mathbb{M}_{J}$. Then $v_{b^{\prime}}^{\mathbb{M}_{J}} \in W_{0}\left(v_{b}\right)$. Hence $v_{b}-v_{b^{\prime}}^{\mathbb{M}_{J}} \in \sum_{\alpha \in \Delta} \mathbb{Q}_{\geq 0} \alpha^{\vee}$.

By assumption, $\lambda^{\diamond}-v_{b} \in \sum_{\alpha \in \Delta} \mathbb{Q}_{>0} \alpha^{\vee}$. Thus $\lambda^{\diamond}-v_{b^{\prime}}^{\mathbb{M}_{J}} \in \sum_{\alpha \in \Delta} \mathbb{Q}_{>0} \alpha^{\vee}$ and cannot be written as a linear combination of the coroots in $\mathbb{M}_{J}$. Therefore $\kappa_{\mathbb{M}_{J}}\left(\sigma^{-1}(y) w y^{-1}\right) \neq \kappa_{\mathbb{M}_{J}}\left(b^{\prime}\right)$. By Theorem 6.3, $X_{w}(b)=\emptyset$.

\subsection{Dimension formula}

Suppose that $\kappa(w)=\kappa(b)$ and $\operatorname{supp}_{\sigma}\left(\eta_{\sigma}(w)\right)=\mathbb{S}$. By Theorem 5.5, there exist a dominant coweight $\gamma \geq_{\mathbb{Z}} \lambda_{w}^{b b}$ and $a \in W_{0}$ with $\operatorname{supp}_{\sigma}(a)=\mathbb{S}$ such that

$$
w \Rightarrow_{\sigma} a t^{\gamma} .
$$

By our assumption, $\gamma^{\diamond} \geq\left(\lambda_{w}^{b b}\right)^{\diamond} \geq v_{b}$. By Theorem 4.2, [ $\left.\dot{t}^{\gamma}\right]=\left[b_{a t^{\gamma}}\right]$. Since $\kappa(w)=\kappa\left(t^{\gamma}\right)=\kappa(b)$, we have $[b] \leq\left[\dot{t}^{\gamma}\right]$.

\footnotetext{
${ }^{1}$ There we assume that $[b]$ is basic. In fact, the assumption is required in [GHN15, Propositon 3.5.1 \& Remark 3.6.2], but it is not needed in [GHN15, Corollary 3.6.1].
} 
By [He15, Theorem 2.27], $X_{a t^{\gamma}}(\dot{\tau}) \neq \emptyset$, where $\tau \in \Omega$ with $\kappa(w)=\kappa\left(t^{\gamma}\right)=\kappa(\tau)$. Since $\kappa(w)=\kappa(b)$, we have $[\dot{\tau}] \leq[b]$.

By Theorem 4.2, $a t^{\gamma}$ is a cordial element. Hence by Theorem 4.1(1), $X_{a t}(b) \neq \emptyset$, and by Theorem 4.1(2), $\operatorname{dim} X_{a t^{\gamma}}(b)=d_{a t}(b)$.

So by the definition of $\Rightarrow_{\sigma}$, we have $X_{w}(b) \neq \emptyset$ and

$$
\operatorname{dim} X_{w}(b)-d_{w}(b) \geq \operatorname{dim} X_{a t^{\gamma}}(b)-d_{a t}(b)=0 .
$$

Hence $\operatorname{dim} X_{w}(b) \geq d_{w}(b)$. On the other hand, by Theorem 2.1, $\operatorname{dim} X_{w}(b) \leq d_{w}(b)$. So $\operatorname{dim} X_{w}(b)=$ $d_{w}(b)$.

\subsection{Some remarks on the condition $\left(\lambda_{w}^{b b}\right)^{\diamond} \geq v_{b}$}

We first consider the case where $[b]$ is basic. In this case, the condition $\left(\lambda_{w}^{b b}\right)^{\diamond} \geq v_{b}$ follows directly from the condition $\kappa(b)=\kappa(w)$.

Now we consider nonbasic $[b]$. Suppose that $\lambda_{w}^{\diamond} \geq v_{b}+2 \rho^{\vee}$. In this case, although $\lambda_{w}-2 \rho^{\vee}$ may not be dominant, its $\sigma$-average is dominant and is larger than or equal to $v_{b}$. By definition, $\lambda_{w}^{b b}-\left(\lambda_{w}-\rho_{J}^{\vee}-\eta_{J}^{\vee}\right) \in \sum_{\alpha \in \Delta} \mathbb{Q}_{\geq 0} \alpha^{\vee}$ for some $J$. Note that $2 \rho^{\vee}-\rho_{J}^{\vee}-\eta_{J}^{\vee} \in \sum_{\alpha \in \Delta} \mathbb{Q}_{\geq 0} \alpha^{\vee}$. We have $\lambda_{w}^{b b}-\left(\lambda_{w}-2 \rho^{\vee}\right) \in \sum_{\alpha \in \Delta} \mathbb{Q}_{\geq 0} \alpha^{\vee}$. Hence $\left(\lambda_{w}^{b b}\right)^{\diamond} \geq \lambda_{w}^{\diamond}-2 \rho^{\vee} \geq v_{b}$. It is also easy to see that $\lambda_{w}^{\diamond}-v_{b} \in \sum_{\alpha \in \Delta} \mathbb{Q}_{>0} \alpha^{\vee}$.

In particular, if $\lambda_{w}=n \omega^{\vee}$, where $\omega^{\vee}$ is a fundamental coweight and $n \gg 0$ with respect to [b], then $\lambda_{w}^{\diamond} \geq v_{b}+2 \rho^{\vee}$, and hence the condition $\left(\lambda_{w}^{b b}\right)^{\diamond} \geq v_{b}$ is satisfied in this case.

Corollary 6.4. Suppose that $\mathbf{G}$ is simple and quasi-split over F. Set $b \in \breve{G}$ and $w \in \tilde{W}$ such that wa is in a shrunken Weyl chamber. Suppose that $\lambda_{w}^{\diamond} \geq v_{b}+2 \rho^{\vee}$. Then $X_{w}(b) \neq \emptyset$ if and only if $\kappa(b)=\kappa(w)$ and $\operatorname{supp}_{\sigma}\left(\eta_{\sigma}(w)\right)=\mathbb{S}$. In this case, $\operatorname{dim} X_{w}(b)=d_{w}(b)$.

\subsection{A side remark}

By Theorem 4.4, if $X_{w}(b) \neq \emptyset$, then $\kappa(b)=\kappa(w)$ and $v_{b} \leq \lambda_{w}^{\diamond}$.

Set $w \in \tilde{W}$ such that $w \mathfrak{a}$ is in a shrunken Weyl chamber. If $\operatorname{supp}_{\sigma}\left(\eta_{\sigma}(w)\right)=\mathbb{S}$, then Theorem 6.1 describes the nonemptiness pattern and the dimension formula of $X_{w}(b)$ for most of the $\sigma$-conjugacy classes $[b]$ with $\kappa(b)=\kappa(w)$ and $v_{b} \leq \lambda_{w}^{\diamond}$.

If $\operatorname{supp}_{\sigma}\left(\eta_{\sigma}(w)\right)=J \varsubsetneqq \mathbb{S}$, then by [GHN15, Lemma 3.6.3] $w$ is a $\left(J, \sigma^{-1}(y), \sigma\right)$-alcove element for some $y \in W_{0}$. Then the Hodge-Newton decomposition (see [GHKR10, Theorem 2.1.4] for the split group and [GHN15, Propositon 2.5.1 \& Theorem 3.3.1] in general) reduces the study of $X_{w}(b)$ to the study of a suitable affine Deligne-Lusztig variety associated to the Levi subgroup $\mathbb{M}_{J}$. One may apply Theorem 6.1 to the latter. In this way, one also obtains an explicit description of the nonemptiness pattern and the dimension formula of $X_{w}(b)$ for most of the $\sigma$-conjugacy classes $[b]$ with $\kappa(b)=\kappa(w)$ and $v_{b} \leq \lambda_{w}^{\diamond}$.

Acknowledgments. We thank S. Nie, E. Viehmann and Q. Yu for helpful discussions. This paper was written during a visit to H. Bao at NUS. We thank the excellent working environment there. We also thank U. Görtz, S. Nie and E. Viehmann for useful comments on a previous version of the paper. Finally, we thank the referees for their helpful suggestions.

The research was partially supported by Hong Kong RGC grant 14300220, by funds connected with the Choh-Ming Chair at CUHK, and by the Xplorer prize. 


\section{References}

[BS17] B. Bhatt and P. Scholze, 'Projectivity of the Witt vector Grassmannian', Invent. Math. 209 (2017), $329-423$.

[BT72] F. Bruhat and J. Tits, 'Groupes rèductifs sur un corps local, I', Publ. Math. Inst. Hautes Études Sci. 41 (1972), 5-276.

[DL76] P. Deligne and G. Lusztig, 'Representations of reductive groups over finite fields', Ann. of Math. (2) 103(1) (1976), $103-161$

[Ga10] Q. Gashi, 'On a conjecture of Kottwitz and Rapoport', Ann. Sci. Éc. Norm. Supér. (4) 43(6) (2010), 1017-1038.

[GHKR10] U. Görtz, T. Haines, R. Kottwitz and D. Reuman, 'Affine Deligne-Lusztig varieties in affine flag varieties', Compos. Math.146 (2010), 1339-1382.

[GH10] U. Görtz and X. He, 'Dimension of affine Deligne-Lusztig varieties in affine flag varieties', Doc. Math. 15 (2010), $1009-1028$.

[GHN15] U. Görtz, X. He and S. Nie, ' $P$-alcoves and nonemptiness of affine Deligne-Lusztig varieties', Ann. Sci. Èc. Norm. Supér. (4) 48 (2015), 647-665.

[GW10] U. Görtz and T. Wedhorn, Algebraic Geometry I. Schemes with Examples and Exercises, Advanced Lectures in Mathematics ( Vieweg + Teubner, Wiesbaden, Germany, 2010).

[HV11] U. Hartl and E. Viehmann, 'The Newton stratification on deformations of local G-shtukas', J. Reine Angew. Math. 656 (2011), 87-129.

[He09] X. He, 'A subalgebra of 0-Hecke algebra', J. Algebra 322 (2009), 4030-4039.

[He14] X. He, 'Geometric and homological properties of affine Deligne-Lusztig varieties', Ann. of Math. (2) 179 (2014), 367-404.

[He15] X. He, 'Hecke algebras and p-adic groups', in Current Developments in Mathematics 2015 (International Press, Somerville, MA, 2016), 73-135.

[HN14] X. He and S. Nie, 'Minimal length elements of extended affine Weyl group', Compos. Math. 150(11) (2014), 1903-1927.

[HR17] X. He and M. Rapoport, 'Stratifications in the reduction of Shimura varieties', Manuscripta Math. 152 (2017), 317-343.

[HY12] X. He and Z. Yang, 'Elements with finite Coxeter part in an affine Weyl group', J. Algebra 372 (2012), $204-210$.

[HY21] Q. Yu and X. He, 'Dimension formula of the affine Deligne-Lusztig variety $X(\mu, b)$ ', Math. Ann. 379 (2021), 1747-1765.

[IM65] N. Iwahori and H. Matsumoto, 'On some Bruhat decomposition and the structure of the Hecke rings of $p$-adic Chevalley groups', Publ. Math. Inst. Hautes Études Sci. 25 (1965), 5-48.

[Ko85] R. Kottwitz, 'Isocrystals with additional structure', Compos. Math. 56 (1985), 201-220.

[Ko97] R. Kottwitz, 'Isocrystals with additional structure. II', Compos. Math. 109 (1997), 255-339.

[MST19] E. Milićević, P. Schwer and A. Thomas, Dimensions of Affine Deligne-Lusztig Varieties: A New Approach via Labeled Folded Alcove Walks and Root Operators, Memoirs of the American Mathematical Society, vol. 261 (2019), no. 1260.

[MV20] E. Milićević and E. Viehmann, 'Generic Newton points and the Newton poset in Iwahori-double cosets', Forum Math. Sigma 8 (2020), E50.

[Ra05] M. Rapoport, 'A guide to the reduction modulo $p$ of Shimura varieties', Astérisque 298 (2005), 271-318.

[Vi14] E. Viehmann, 'Truncations of level 1 of elements in the loop group of a reductive group', Ann. of Math. (2) 179(3) (2014), 1009-1040.

[Zu17] X. Zhu, 'Affine Grassmannians and the geometric Satake in mixed characteristic', Ann. of Math. (2) 185 (2017), 403-492. 